\title{
MATTI SOITTOLA
}

\section{Remarks on DOL growth sequences}

Revue française d'automatique, informatique, recherche opérationnelle. Informatique théorique, tome $10, \mathrm{n}^{\circ} \mathrm{R} 3$ (1976), p. 23-34.

<http://www.numdam.org/item?id=ITA_1976_10_3_23_0>

(C) AFCET, 1976, tous droits réservés.

L'accès aux archives de la revue « Revue française d'automatique, informatique, recherche opérationnelle. Informatique théorique » implique l'accord avec les conditions générales d'utilisation (http://www.numdam.org/legal. php). Toute utilisation commerciale ou impression systématique est constitutive d'une infraction pénale. Toute copie ou impression de ce fichier doit contenir la présente mention de copyright.

\section{Numdam}

Article numérisé dans le cadre du programme

Numérisation de documents anciens mathématiques

http://www.numdam.org/ 


\title{
REMARKS ON DOL GROWTH SEQUENCES ( $\left.{ }^{*}\right)$
}

\author{
by Matti SoITTOLA $\left(^{1}\right)$
}

Communicated by J. Berstel

\begin{abstract}
Two theorems are given characterizing the position of DOL and PDOL growth sequences among $N$-rational sequences.
\end{abstract}

\section{INTRODUCTION}

A DOL system or a deterministic context-independent Lindenmayer system consists of an initial word $\omega$ and a set of productions $x \rightarrow \delta(x)$ which give for any letter $x$ and thus also for any word a unique successor. The growth sequence of a DOL system is the sequence formed by the lengths of the words $\omega, \delta(\omega)$, $\delta^{2}(\omega), \ldots$ DOL sequences have been investigated e. g. in Paz and Salomaa [6], Salomaa [8], Vitànyi [10], Ruohonen [7] and Karhumäki [4].

A sequence $\left(r_{n}\right)$ is called $N$-rational if it can be represented in the form $r_{n}=P M^{n} Q$ where $P$ is a row vector, $M$ is a square matrix, $Q$ is a column vector and the entries of $P, \mathrm{M}$ and $Q$ are natural numbers. (The name $N$-rational comes from the general theory of rational series founded by M. P. Schützenberger.) Now it is easy to see that a DOL sequence is $N$-rational; in fact it has a representation $P M^{n} Q$ where $Q$ consists merely of ones.

If a DOL sequence is not terminating, i. e. if $r_{n} \neq 0$ for every $n$, and if $L$ is the largest of the lengths of the words $\delta(x)$ then obviously $r_{n+1} / r_{n} \leqq L$ for every $n$. If the system under consideration is such that $\delta(x)$ is always a non-empty word then this system is called a PDOL system and its growth sequence is called a PDOL sequence. Obviously a PDOL sequence is nondecreasing.

The goal of this paper is to illustrate the position of DOL and PDOL sequences among $N$-rational sequences. It will be seen that the satisfaction of an inequality $r_{n+1} / r_{n} \leqq L$ is characteristic for DOL sequences. Further it will be seen that it is not the non-negativity but the $N$-rationality of the sequence $\left(r_{n+1}-r_{n}\right)$ that makes a DOL sequence to be a PDOL sequence.

\section{PRELIMINARIES}

A DOL system is at triple $G=(X, \delta, \omega)$ where $X=\left\{x_{1}, \ldots, x_{k}\right\}$ is an alphabet, $\delta$ is an endomorphism of the free monoid $X^{*}$ and $\omega \in X^{*}$. The mapping $\delta$ is usually given by writing the oductions $x_{i} \rightarrow \delta\left(x_{i}\right)$ and the

(*) Reçu février 1976.

(') Department of Mathematics, University of Turku, Finland.

Revue Française d'Automatique, Informatique et Recherche Opérationnelle, décembre 1976. 
word $\omega$ is called the axiom. If $\delta\left(x_{i}\right) \neq \lambda$ for each $i$ then $G$ is called a PDOL system. The function

$$
f_{G}(n)=\lg \left(\delta^{n}(\omega)\right)
$$

where $\mathrm{lg}$ means word length is called the growth function of $G$.

A pair $(X, \delta)$ where $X$ and $\delta$ are as above is called a DOL scheme.

Introducing the axiom vector

$$
P=\left(\lg _{1}(\omega), \ldots, \lg _{k}(\omega)\right)
$$

and the growth matrix

$$
M=\left(\begin{array}{ccc}
\lg _{1}\left(\delta\left(x_{1}\right)\right) & \ldots & \lg _{k}\left(\delta\left(x_{1}\right)\right) \\
\cdot & \ldots & \cdot \\
\lg _{1}\left(\delta\left(x_{k}\right)\right) & \ldots & \lg _{k}\left(\delta\left(x_{k}\right)\right)
\end{array}\right)
$$

of $G$ (here $\lg _{j}$ denotes the number of letters $x_{j}$ ) we obtain

$$
\left(\lg _{1}\left(\delta^{n}(\omega)\right), \ldots, \lg _{k}\left(\delta^{n}(\omega)\right)\right)=P M^{n}
$$

and

$$
f_{G}(n)=P M^{n}(1, \ldots, 1)^{T} .
$$

A sequence $(r(n))$ is called $Z$-rational (resp. $N$-rational) if

$$
r(n)=P M^{n} Q=\left(p_{1}, \ldots, p_{k}\right)\left(\begin{array}{ccc}
m_{11} & \ldots & m_{1 k} \\
\cdot & \ldots & \cdot \\
m_{k 1} & \ldots & m_{k k}
\end{array}\right)^{n}\left(\begin{array}{c}
q_{1} \\
\cdot \\
q_{k}
\end{array}\right)
$$

where all the entries are integers (resp. non-negative integers). If now $G$ is a DOL system (resp. a PDOL system) then by the above $\left(f_{G}(n)\right)$ is a special $N$-rational sequence called a DOL sequence (resp. a PDOL sequence).

It is known (Schützenberger [9]) that a sequence $(r(n))$ is $Z$-rational ( $N$-rational) iff the series $\sum r(n) x^{n}$ is $Z$-rational ( $N$-rational). If now $\sum r(n) x^{n}$ is a non-polynomial $N$-rational series then a theorem of Berstel [1] concerning its poles tells the following: there are a natural number $p$, algebraic numbers $A, A_{1}, \ldots, A_{s}\left(A>0,\left|A_{j}\right|<A, s \geqq 0\right)$ and polynomials $H_{0}, \ldots, H_{p-1}, h_{1}, \ldots$, $h_{s}$ such that

$$
r(i+n p)=H_{i}(i+n p) A^{i+n p}+\sum_{j=1}^{s} h_{j}(i+n p) A_{j}^{i+n p}
$$

for large values of $n(i=0, \ldots, p-1)$. In the case of a DOL sequence the polynomials $H_{i}$ must have a common degree $l$ because the quotients $r(n+1) / r(n)$ are bounded from above. We shall say that $(r(n))$ has the growth order $n^{l} A^{n}$. 


\section{THE PDOL SEQUENCES}

Lemma 1: If $(f(n))=\left(P M^{n} Q\right)$ is an $N$-rational sequence and $P$ has positive entries then $(f(n))$ is a DOL sequence.

Proof: Let $G=\left(\left\{x_{1}, \ldots, x_{k}\right\}, \delta, \omega\right)$ be a DOL system with axiom vector $Q^{T}$ and growth matrix $M^{T}$. Define $G^{\prime}=\left(\left\{x_{1}, \ldots, x_{k}, x\right\}, \delta^{\prime}, \omega^{\prime}\right)$ where

$$
\begin{gathered}
\delta^{\prime}\left(x_{i}\right)=\delta\left(x_{i}\right) x^{\lg _{1}\left(\delta\left(x_{i}\right)\right)\left(p_{1}-1\right)+\ldots+\lg \left(\delta\left(x_{i}\right)\right)\left(p_{k}-1\right)}, \\
\delta^{\prime}(x)=\lambda, \\
\omega^{\prime}=\omega x^{\lg (\omega)\left(p_{1}-1\right)+\ldots+\lg _{k}(\omega)\left(p_{k}-1\right)} .
\end{gathered}
$$

Then obviously $f(n)=Q^{T}\left(M^{T}\right)^{n} P^{T}=f_{\mathrm{G}}(n)$.

THEOREM 1: Let $(r(n))$ be an $N$-rational sequence. Then we can find natural numbers $m$ and $p$ and DOL sequences $\left(d_{0}(n)\right), \ldots,\left(d_{p-1}(n)\right)$ such that

$$
r(m+i+n p)=d_{i}(n) \quad(i=0, \ldots, p-1) .
$$

Proof: Let $r(n)=P M^{n} Q$ and let $G=(X, \delta, \omega)$ be a DOL system with axiom vector $P$ and growth matrix $M$. Denote by $X_{n}$ the set of letters occurring in $\delta^{n}(\omega)$. Then we can find numbers $m$ and $p$ such that $X_{n+i}=X_{m+i+n p}$. We may of course suppose that no $y_{n}$ is empty.

Introduce now the DOL systems

$$
G_{i}=\left(X_{m+i}, \delta^{p}, \delta^{m+i}(\omega)\right) \quad(i=0, \ldots, p-1)
$$

whose axiom vectors and growth matrices are denoted by $P_{i}$ and $M_{i}$. Then obviously

$$
r(m+i+n p)=P_{i} M_{i}^{n} Q_{i},
$$

where $Q_{i}$ is composed of those entries of $Q$ corresponding to letters of $X_{m+i}$. By lemma 1 we may define $d_{i}(n)=P_{i} M^{n} Q_{i}$.

THEOREM 2: The following conditions are equivalent for a sequence $(r(n))$ :

(i) $(r(n))$ is a PDOL sequence not identically zero;

(ii) $r(0)$ is a positive integer and the sequence $(s(n))=(r(n+1)-r(n))$ is $N$-rational.

Proof: Suppose (i) holds. If now $(r(n))$ corresponds to a PDOL system $G=\left(\left\{x_{1}, \ldots, x_{k}\right\}, \delta, \omega\right)$ then

$$
s(n)=\sum_{i=1}^{k} \lg _{i}\left(\delta^{n}(\omega)\right)\left(\lg \left(\delta\left(x_{i}\right)\right)-1\right)
$$

and each of the sequences $\left(\lg _{i}\left(\delta^{n}(\omega)\right)\right)(i=1, \ldots, k)$ is $N$-rational. décembre 1976. 
Suppose then that (ii) holds. Write according to theorem 1

$$
s(m+i+n p)=d_{i}(n) \quad(i=0, \ldots, p-1)
$$

where $\left(d_{i}(n)\right)$ corresponds to a system $G_{i}=\left(X_{i}, \delta_{i}, \omega_{i}\right)$. Assuming that the alphabets $X_{i}$ are mutually disjoint we construct the PDOL system $G=(X, \delta, \omega)$ where

and

$$
\begin{gathered}
X=\left(\bigcup_{i=0}^{p-1} \bigcup_{j=0}^{p-1} X_{i}^{(j)}\right) \cup\{y\}, \\
\omega=\omega_{0}^{(p-1)} \omega_{1}^{(p-2)} \ldots \omega_{p-2}^{(1)} \omega_{p-1}^{(0)}
\end{gathered}
$$

$$
\begin{gathered}
x^{(j)} \rightarrow x^{(j+1)} \quad \text { when } \quad x^{(j)} \in X_{i}^{(j)}, j<p-1, \\
x^{(p-1)} \rightarrow \delta_{i}(x)^{(0)} y \quad \text { when } x^{(p-1)} \in X_{i}^{(p-1)}, \\
y \rightarrow y .
\end{gathered}
$$

Disregarding the non-commutativity of letters we may write

$$
\begin{aligned}
\omega & \rightarrow \delta_{0}\left(\omega_{0}\right)^{(0)} y^{\lg \left(\omega_{0}\right)} \omega_{1}^{(p-1)} \ldots \omega_{p-2}^{(2)} \omega_{p-1}^{(1)} \\
& \rightarrow \delta_{0}\left(\omega_{0}\right)^{(1)} y^{l g\left(\omega_{0}\right)} \delta_{1}\left(\omega_{1}\right)^{(0)} y^{\lg \left(\omega_{1}\right)} \ldots \omega_{p-1}^{(2)} \\
& \rightarrow \ldots
\end{aligned}
$$

Thus

$$
\begin{aligned}
r(n)= & (r(0)+s(0)+\ldots+s(m-1)) \\
& +(s(m)+\ldots+s(m+p-1)) \\
& +s(m+p)+\ldots+s(n-1) \\
= & (r(0)+s(0)+\ldots+s(m-1))+f_{G}(n-m-p),
\end{aligned}
$$

when $n \geqq m+p$. It is now easy to extend $G$ to a PDOL system $G^{\prime}$ for which $f_{\mathrm{G} \cdot}(n)=r(n)$.

Lemma 2: Let $(r(n))$ be a Z-rational sequence. Then for any large natural number $R$ the sequence defined by

$$
\begin{gathered}
d(0)=d(1)=1, \\
d(2 n)=R^{2 n}-r(2 n), \\
d(2 n+1)=R^{2 n}-r(2 n+1) \quad(n>0)
\end{gathered}
$$

is a DOL sequence.

Proof: Let at first $(r(n))$ be a DOL sequence corresponding to the system $G=(X, \delta, \omega)$. Construct the system $H=\left(X \cup \bar{X} \cup \overline{\bar{X}} \cup\{a, b\}, \delta^{\prime}, \Omega\right)$ where

$$
\Omega=a^{R^{2}-2 r(2)-r(3)} \delta^{2}(\omega) \overline{\delta^{3}(\omega)}
$$


and

$$
\begin{gathered}
x \rightarrow b \overline{\bar{x}}, \quad \bar{x} \rightarrow \lambda, \quad a \rightarrow b, \quad b \rightarrow a^{R^{2}}, \\
\overline{\bar{x}} \rightarrow a^{R^{2}(1+\lg (\delta(x)))-2 \lg \left(\delta^{2}(x)\right)-\lg \left(\delta^{3}(x)\right)} \delta^{2}(x) \overline{\delta^{3}(x) .}
\end{gathered}
$$

It is immediately seen that $f_{H}(n)=d(n+2)$.

This implies our lemma because of the following. It is known that every $Z$-rational sequence is the difference of two $N$-rational sequences (see [9] remark 2 or [2] p. 218). Furthermore, every $N$-rational sequence is the difference of two DOL sequences for

$$
P M^{n} Q=(P+(1, \ldots, 1)) M^{n} Q-(1, \ldots, 1) M^{n} Q .
$$

Hence every $Z$-rational sequence can be written as the difference of two DOL sequences.

TheOrem 3: Not every increasing DOL sequence is a PDOL sequence.

Proof: Using lemma 2 we see that when $R$ is a large natural number then the sequence $(d(n))$ where

$$
\begin{gathered}
d(0)=d(1)=1, \\
d(2 n)=R^{2 n}, \\
d(2 n+1)=R^{2 n}+\left(\operatorname{Re}(3+4 i)^{2 n+1}\right)^{2} \quad(n>0)
\end{gathered}
$$

is an increasing DOL sequence. Now

$$
\operatorname{Re}(3+4 i)^{n}=\cos 2 \pi n \alpha \cdot 5^{n},
$$

where $\alpha$ is irrational because for every positive $n \operatorname{Im}(3+4 \mathrm{i})^{n}<4(\bmod 5)$. The theorem of Berstel [1] then implies that the sequence $(d(2 n+1)-d(2 n))$ cannot be $N$-rational. Therefore $(d(n))$ is not a PDOL sequence.

Note: Let $(d(n))=\left(P M^{n} \mathrm{Q}\right)$ be a DOL sequence. By lemma 4 below we have $M^{m+p} \geqq M^{m}$ for some integers $m$ and $p(p>0)$. But then each of the sequences

$$
(d(m+i+(n+1) p)-d(m+i+n p)) \quad(i=0, \ldots, p-1)
$$

is a DOL sequence and so the sequences

$$
(d(m+i+n p)) \quad(i=0, \ldots, p-1)
$$

are PDOL sequences. This result also appears in [5] (proof of th. 4.12).

\section{THE DOL SEQUENCES}

Let $G=\left(\left\{x_{1}, \ldots, x_{k}\right\}, \delta\right)$ be a DOL scheme such that for any letter $x_{i}$ :

$$
\lg \left(\delta^{n}\left(x_{i}\right)\right) \sim g_{i} A^{n} \quad \text { as } n \rightarrow \infty \quad\left(g_{i}>0, A \geqq 1\right) .
$$

décembre 1976. 
M. SOITTOLA

If $w \in\left\{x_{1}, \ldots, x_{k}\right\}^{+}$then the number

$$
g(w)=\lg _{1}(w) g_{1}+\ldots+\lg _{k}(w) g_{k}
$$

is called the growth coefficient of $w$.

Suppose we have $p$ DOL schemes $G_{i}=\left(X_{i}, \delta_{i}\right)(i=0, \ldots, p-1)$ satisfying the condition of the above definition with a common number $A$. Introduce the infinite alphabet

and define

$$
X=\left\{\left(W_{0}, \ldots, W_{p-1}\right) \mid W_{i} \in X_{i}^{+}\right\}
$$

$$
\partial\left(W_{0}, \ldots, W_{p-1}\right)=\left(\delta_{0} W_{0}, \ldots, \delta_{p-1} W_{p-1}\right)
$$

Define further

$$
\pi\left(W_{0}, \ldots, W_{p-1}\right)=\left(\eta\left(W_{0}\right), \ldots, \eta\left(W_{p-1}\right)\right),
$$

where $\eta$ means Parikh-vector. Take then a fixed element $\left(\omega_{0}, \ldots, \omega_{p-1}\right)$ of $X$ and denote

$$
Y=\left\{\left(W_{0}, \ldots, W_{p-1}\right) \mid \frac{g\left(W_{0}\right)}{g\left(\omega_{0}\right)}=\ldots=\frac{g\left(W_{p-1}\right)}{g\left(\omega_{p-1}\right)}\right\} .
$$

Obviously $\left(W_{0}, \ldots, W_{p-1}\right) \in Y$ implies that also $\delta\left(W_{0}, \ldots, W_{p-1}\right) \in Y$.

LEMMA3 : There are vectors $V_{1}, \ldots, V_{J}$ of $\pi(Y)$ such that any vector in $\pi(Y)$ is a sum of these.

Proof: Let $\pi(Y) \subseteq N^{k}$. By the definition of $Y$ there are algebraic numbers $a_{i j}(i=1, \ldots, p \sim 1 ; j=1, \ldots, k)$ such that $\vec{v}=\left(n_{1}, \ldots, n_{k}\right) \in Z^{k}$ is in $\pi(Y)$ iff

and

$$
\ddot{v} \neq 0, \quad \ddot{v} \geqq 0,
$$

$$
a_{i 1} n_{1}+\ldots+a_{i k} n_{k}=0 \quad(i=1, \ldots, p-1) .
$$

Let $F$ be the additive subgroup of $Z^{k}$ defined by the above linear system.

We shall need the following simple lemma (see [3]):

LEMMA 4: Any subset of $N^{k}$ contains only a finite number of minimal vectors (with respect to the natural componentwise ordering).

Let now $\ddot{V}_{1}, \ldots, \bar{V}_{J}$ be the minimal vectors of $\pi(Y)$. If $\bar{V} \in \pi(Y)$ then it has a representation $\bar{V}=\bar{V}_{i}+\bar{U}$ where $\bar{U} \in N^{k}$. But if $\bar{U} \neq \overline{0}$ it is in $\pi(Y)$ because it belongs to $F$. Repeating this process we obtain $\bar{V}$ as a sum of the minimal vectors.

THEOREM 4: Let $G_{i}=\left(X_{i}, \delta_{i}, \omega_{i}\right)(i=0, \ldots, p-1)$ be DOL systems such that if $x_{j} \in X_{i}$ then

$$
\lg \left(\delta_{i}^{n}\left(x_{j}\right)\right) \sim g_{i j} A^{n} \quad \text { as } n \rightarrow \infty \quad\left(g_{i j}>0, A \geqq 1\right) .
$$

Revue Française d'Automatique, Informatique ef Recherche Opiratimnells' 
Then the sequence defined by

$$
d(n p+i)=\lg \left(\delta_{i}^{n}\left(\omega_{i}\right)\right) \quad(n=0,1, \ldots, i=0, \ldots, p-1)
$$

is a DOL sequence.

Proof: Take $p$ copies $X_{i}^{(0)}, \ldots, X_{i}^{(p-1)}$ of each $X_{i}$ and define

$$
Y=\bigcup_{j=0}^{p-1}\left\{\left(W_{0}^{(j)}, \ldots, W_{p-1}^{(j)}\right) \mid W_{i}^{(j)} \in X_{i}^{(j)+}, \quad \frac{g\left(W_{0}\right)}{g\left(\omega_{0}\right)}=\ldots=\frac{g\left(W_{p-1}\right)}{g\left(\omega_{p-1}\right)}\right\} .
$$

Let $V_{1}^{(0)}, \ldots, V_{J}^{(0)}, \ldots, V_{1}^{(p-1)}, \ldots, V_{J}^{(p-1)}$ be elements of $Y$ corresponding to the vectors given by lemma 3 . We may say that any element of $Y$ is a commutative product of these elements.

Introduce the DOL system

$$
G=\left(\left\{V_{1}^{(0)}, \ldots, V_{J}^{(p-1)}\right\} \cup\{y\}, \delta, \omega\right)=(Z \cup\{y\}, \delta, \omega),
$$

where $\delta$ and $\omega$ are as follows:

$\omega$ consists of $\left(\omega_{0}^{(0)}, \ldots, \omega_{p-1}^{(0)}\right)$ written commutatively in the alphabet $Z$ and of so many $y$ 's that $\lg (\omega)$ becomes equal to $\lg \left(\omega_{0}\right)$;

$y$ produces $\lambda$;

when $j<p-1\left(W_{0}^{(i)}, \ldots, W_{p-1}^{(j)}\right)$ produces $\left(W_{0}^{j+1}, \ldots, W_{p-1}^{(j+1)}\right.$ and so many $y$ 's that the length of the produced word will be $\lg \left(W_{j+1}\right)$;

$\left(W_{0}^{(p-1)}, \ldots, W_{p-1}^{(p-1)}\right)$ produces $\left(\delta_{0}\left(W_{0}\right)^{(0)}, \ldots, \delta_{p-1}\left(W_{p-1}\right)^{(0)}\right)$ written commutatively in the alphabet $Z$ and so many $y$ 's that the produced word will have length $\lg \left(\delta_{0}\left(W_{0}\right)\right)$.

Heuristically, derivations in the systems $G_{i}$ are simulated in the components of the letters of $Z$. With the aid of the $p$ copies taken of the alphabets the simulation is delayed to happen only at intervals of $p$ steps. By using the letter $y$ the length of the word $\delta^{i+}{ }^{n} p(\omega)$ is adjusted to be equal to that of $\delta_{i}^{n}\left(\omega_{i}\right)$. This is possible because the components of the letters of $Z$ are nonempty words.

It should be clear now that $(d(n))$ is the growth sequence of $G$.

Let $G=(X, \delta)$ be a DOL scheme which gives a growth of order $n^{l} A^{n}(A \geqq 1, l \geqq 0)$ but does not give a growth of higher order. We divide $X$ into classes $\Sigma, \Sigma_{0}, \ldots, \Sigma_{l}$ as follows: the letters of $\Sigma$ generate a growth having smaller order than $A^{n}$ and the letters of $\Sigma_{i}$ generate a growth of order $n^{i} A^{n}$.

It is clear that a letter of $\Sigma_{i}$ cannot produce letters of $\Sigma_{i+1} \cup \ldots \cup \Sigma_{l}$, it must produce a letter of $\Sigma_{i}$ and it may produce letters of $\Sigma \cup \Sigma_{0} \cup \ldots \cup \Sigma_{i-1}$; a letter of $\Sigma$ may produce only letters of $\Sigma$ or $\lambda$.

Lemma 5: Any letter of $\Sigma_{l}(l>0)$ generates letters of $\Sigma_{l-1}$. If all letters of $\Sigma \cup \Sigma_{0} \cup \ldots \cup \Sigma_{l-1}$ are deleted then the resulting scheme $H$ is such that all letters generate a growth of order $A^{n}$. 
Proof: Suppose $x$ generates in $H$ a growth whose order is at least $n A^{n}$. Then $x$ generates in $G$ in $2 n$ steps a word whose length is at least of the order

$$
n A^{n} n^{l} A^{n}=\left(\begin{array}{l}
1 \\
2
\end{array}\right)^{l+1}(2 n)^{l+1} A^{2 n} \text {. }
$$

This shows that the growth of $x$ in $H$ has the order $A^{n}$ at most.

Assume that $x \in \Sigma_{l}(l>0)$ never generates a letter of $\Sigma_{l-1}$. By the above $\delta^{n}(x)$ contains $O\left(A^{n}\right)$ letters of $\Sigma \cup \Sigma_{0} \cup \ldots \cup \Sigma_{l-2}$ directly produced by letters of $\Sigma_{l}$. But

$$
\sum_{n=0}^{N} A^{n}(N-n)^{l-2} A^{N-n}=A^{N} \sum_{n=0}^{N} n^{l-2}=o\left(N^{l} A^{N}\right)
$$

and so $x$ cannot generate a growth of order $n^{l} A^{n}$. Hence $x$ must generate letters of $\Sigma_{l-1}$.

Assume further that the growth of $x$ in $H$ is majorized by $a^{n}(a<A)$. Because

$$
\sum_{n=0}^{N} a^{n}(N-n)^{l-1} A^{N-n}=a^{N} \sum_{n=0}^{N} n^{l-1}(A / a)^{n}=O\left(N^{l-1} A^{N}\right)
$$

we have a contradiction as above. Thus the growth of $x$ in $H$ has the order $A^{n}$ when $l>0$.

Suppose $x \in \Sigma_{0}$ and the growth of $x$ in $H$ as well as the growth of any letter of $\Sigma$ in $G$ is majorized by $a^{n}(a<A)$. Because

$$
\sum_{n=0}^{N} a^{n} a^{N-n}=o\left(A^{N}\right)
$$

we see that the above result is true also when $l=0$.

THEOREM 5: Let $(r(n))$ be an $N$-rational sequence such that $r(n) \neq 0$ for every $n$ and the quotient $r(n+1) / r(n)$ remains bounded. Then $(r(n))$ is a DOL sequence.

Proof: We know that there are numbers $m$ and $p$ and DOL sequences $\left(d_{0}(n)\right), \ldots,\left(d_{p-1}(n)\right)$ such that

$$
r(m+i+n p)=d_{i}(n) .
$$

By our assumption all these sequences have the same order of growth. We may suppose that it is of the form $n^{l} A^{n}(A>1)$ for the polynomial case is covered by a theorem of Ruohonen [7].

Let $G_{i}=\left(X_{i}, \delta_{i}, \omega_{i}\right)$ be a DOL system corresponding to the sequence $\left(d_{i}(n)\right)$. Write $X_{i}=\Sigma_{i} \cup \Sigma_{i 0} \cup \ldots \cup \Sigma_{i l}$ as before and denote by $H_{i j}$ the DOL schema obtained from $\left(X_{i}, \delta_{i}\right)$ by deleting all letters except those of $\Sigma_{i j}$. 
Suppose $W \in \Sigma_{i 0}^{+}$and $w \in \Sigma_{i}^{*}$. If $W$ generates $V v\left(V \in \Sigma_{i 0}^{+}, v \in \Sigma_{i}^{*}\right)$ and $w$ generates $u$ in $k$ steps then there are constants $N, M$ and $L$ (independent of $k$ and $i$ ) such that

$$
\begin{aligned}
\lg (V) & \geqq N A^{k} \lg (W), \\
\lg (v) & \leqq M A^{k} \lg (W), \\
\lg (u) & \leqq L a^{k} \lg (w) \quad(a<A) .
\end{aligned}
$$

We now see that if $\lg (w) / \lg (W) \leqq \alpha M / N(\alpha \geqq 2, \alpha M / N$ integer $)$ and if $k$ is so large that $(L / N)(a / A)^{k} \leqq 1 / 2$ then

$$
\begin{aligned}
\lg (u v) / \lg (V) & \leqq M / N+(L / N)(a / A)^{k}(\lg (w) / \lg (W)) \\
& \leqq M / N+(1-1 / \alpha) \alpha M / N=\alpha M / N
\end{aligned}
$$

too.

By taking a multiple of $p$, if necessary, we may suppose that the following three conditions hold:

(i) any growth in $H_{i j}(i=0, \ldots, p-1 ; j=0, \ldots, l)$ is asymptotically equal to constant times $A^{n}$;

(ii) any letter of $\Sigma_{i j}(i=0, \ldots, p-1 ; j=0, \ldots, l)$ produces in a step letters of all the alphabets $\Sigma_{i, j-1}, \ldots, \Sigma_{i, 0}$;

(iii) the equation (1) holds with $k=1$.

Moreover, by increasing $m$ we obtain the following situation:

(iv) the axiom of $G_{i}$ contains letters of all the alphabets $\Sigma_{i, 0}, \ldots, \Sigma_{i l}$.

We are now ready to give an induction proof showing that the sequence $(s(n))=(r(n-m))$ is a DOL sequence. This immediately implies our theorem.

If $l=0$ we at first neglect all letters of the $\Sigma_{i}$ 's and construct a system just as in the proof of theorem 4 . Then we take $p$ copies $x^{(0)}, \ldots, x^{(p-1)}$ of each neglected letter $x$ and join these copies to the components of the letters of $Z$ so that the original systems $G_{i}$ become simulated. Condition (iii) assures that this can be done. At the same time we add $y$ 's so that the right lengths are obtained.

When taking the induction step we at first delete all letters of the alphabets $\Sigma_{i} \cup \Sigma_{i 0} \cup \ldots \cup \Sigma_{i, l-1}$ and construct a system according to theorem 4 . By conditions (ii) and (iv) the letters of this system as well as the p-tuple $\left(\omega_{0}, \ldots, \omega_{p-1}\right)$ give axioms for systems whose existence is guaranteed by the induction hypothesis.

Example: Let

$$
G_{0}=\left(\{A, B, C\}, \delta_{0}, A B\right)
$$

where

$$
A \rightarrow A^{4} B, \quad B \rightarrow B^{4} b, \quad b \rightarrow b
$$

décembre 1976. 
and

$$
G_{1}=\left(\{C, D, E, F\}, \delta_{1}, C D\right)
$$

where

$$
C \rightarrow C^{4} D, \quad D \rightarrow D^{2} E^{3}, \quad E \rightarrow E^{2} F^{4}, \quad F \rightarrow D F .
$$

The common order of growth is $n .4^{n}$ and

$$
\begin{array}{ccc}
\Sigma_{0}=\{b\}, \quad \Sigma_{00}=\{B\}, & \Sigma_{01}=\{A\}, \\
\Sigma_{1}=\varnothing, & \Sigma_{10}=\{D, E, F\}, & \Sigma_{11}=\{C\} .
\end{array}
$$

The procedures described in the preceding proof yield e. g. the following system: The axiom is

$$
\left(A^{(0)}, C^{(0)}\right)\left(B^{(0)}, D^{(0)}\right)
$$

and the productions are

$$
\begin{gathered}
\left(A^{(0)}, C^{(0)}\right) \rightarrow\left(A^{(1)}, C^{(1)}\right), \\
\left(A^{(1)}, C^{(1)}\right) \rightarrow\left(A^{(0)}, C^{(0)}\right)^{4}\left(B^{(0)}, D^{(0)}\right), \\
\left(B^{(0)}, D^{(0)}\right) \rightarrow\left(B^{(1)}, D^{(1)}\right), \\
\left(B^{(1)}, D^{(1)}\right) \rightarrow\left(B^{(0)} b^{(0)}, D^{(0)}\right)\left(B^{(0)}, D^{(0)}\right)\left(B^{(0) 2}, E^{(0) 3}\right) y^{2} . \\
\left(B^{(0) 2}, E^{(0) 3}\right) \rightarrow\left(B^{(1) 2}, E^{(1) 3}\right) y^{2}, \\
\left(B^{(1) 2}, E^{(1) 3}\right) \rightarrow\left(B^{(0) 2} b^{(0)}, E^{(0) 3}\right)^{2}\left(B^{(0)}, F^{(0) 3}\right)^{4} y^{4}, \\
\left(B^{(0)}, F^{(0) 3}\right) \rightarrow\left(B^{(1)}, F^{(1) 3}\right) y^{2}, \\
\left(B^{(1)}, F^{(1) 3}\right) \rightarrow\left(B^{(0)} b^{(0)}, F^{(0) 3}\right)\left(B^{(0)}, D^{(0)}\right)^{3} y, \\
\left(B^{(0)} b^{(0)}, D^{(0)}\right) \rightarrow\left(B^{(1)} b^{(1)}, D^{(1)}\right), \\
\left(B^{(1)} b^{(1)}, D^{(1)}\right) \rightarrow\left(B^{(0)} b^{(0)}, D^{(0)}\right)^{2}\left(B^{(0) 2}, E^{(0) 3}\right) y^{3}, \\
\left(B^{(0) 2} b^{(0)}, E^{(0) 3}\right) \rightarrow\left(B^{(1) 2} b^{(1)}, E^{(1) 3}\right) y^{2}, \\
\left(B^{(1) 2} b^{(1)}, E^{(1) 3}\right) \rightarrow\left(B^{(0) 2} b^{(0)}, E^{(0) 3}\right)^{2}\left(B^{(0)} b^{(0)}, F^{(0) 3}\right)\left(B^{(0)}, F^{(0) 3}\right)^{3} y^{5}, \\
\left(B^{(0)} b^{(0)}, F^{(0) 3}\right) \rightarrow\left(B^{(1)} b^{(1)}, F^{(1) 3}\right) y^{2}, \\
\left(B^{(1)} b^{(1)}, F^{(1) 3}\right) \rightarrow\left(B^{(0)} b^{(0)}, F^{(0) 3}\right)\left(B^{(0)} b^{(0)}, D^{(0)}\right)\left(B^{(0)}, D^{(0)}\right)^{2} y^{2}, \\
y \rightarrow \lambda .
\end{gathered}
$$

Note: Let $(d(n))$ be a DOL sequence such that the rational function $\sum d(n) x_{n}$ is not a polynomial. Then it is easy to see that the growth order of $(d(n))$ is $n^{l} A^{n}$ where $1 / \mathrm{A}$ is the smallest positive pole of $\sum d(n) x^{n}$ and $l+1$ 
is its multiplicity. This enables us to effectively compare the growth orders of two DOL sequences; we describe the method briefly in general form.

Given integer polynomials $q_{1}(x), \ldots, q_{p}(x)$ we can (using symmetric polynomials) construct a polynomial $Q(x)$ such that any difference of two zeros of $q(x)=q_{1}(x) \ldots q_{p}(x)$ is a zero of $Q(x)$. Thus we can give a positive number $\gamma$ such that if $z_{1}$ and $z_{2}$ are zeros of $q(x)$ then either $z_{1}=z_{2}$ or $\left|z_{1}-z_{2}\right|>\gamma$.

The polynomial

$$
Q_{i}(x)=\frac{q_{i}(x)}{g \cdot c \cdot d \cdot\left(q_{i}(x), q_{:}^{\prime}(x)\right)}
$$

has simple zeros which are the same as those of $q_{i}(x)$. Therefore we can compare the real roots of the polynomials $q_{i}(x)$ by examining the sign changes of the polynomials $Q_{i}(x)$.

Because $\alpha$ is a $k$-fold zero of $q_{i}(x)$ iff it is a zero of $q_{i}(x), q_{i}^{\prime}(x), \ldots, q_{i}^{(k-1)}(x)$ but not a zero of $q^{(k)}(x)$ it is possible to determine the multiplicity of any real zero of $q_{i}(x)$.

An $N$-rational sequence $(r(n))$ is by theorem 5 a DOL sequence iff one of the following conditions holds:

(i) there is a natural number $L$ such that $r(n)>0$ when $n \leqq L$ and $r(n)=0$ when $n>L$;

(ii) every $r(n)$ is positive and the DOL sequences given by theorem 1 have the same growth order.

Hence it is possible to decide whether or not a given $N$-rational sequence is a DOL sequence.

\section{ACKNOWLEDGEMENT}

The author is grateful to Mr. J. Karhumäki for the useful conments he made during the preparation of this paper.

\section{REFERENCES}

1. J. Berstel, Sur la densité asymptotique de langages formels, in Automata, languages and programming (ed. M. NivAT), North-Holland, 1973, pp. 345-358.

2. S. Eilenberg, Automata, Languages and Machines, Vol. A, Academic Press, 1974.

3. S. EILENBerg and N. P. SchüTZENBERger, Rational Sets in Commutative Monoids, J. Algebra, vol. 13, 1969, pp. 173-191.

4. J. KARHUMÄKI, Two Theorems Concerning Recognizable $N$-Subsets of $\sigma^{*}$, to appear in Theoretical Computer Science.

5. M. Nielsen, G. Rozenberg, A. SalomaA and S. Skyum, Nonterminals, Homomorphisms and Codings in Different Variations of OL Systems Acta Informat., vol. 4, 1974, pp. 87-106.

décembre 1976. 
6. A. Paz and A. SalomaA, Integral Sequential Word Functions and Growth Equivalence of Lindenmayer Systems, Information and Control, vol. 23, 1973, pp. 313343.

7. K. Ruohonen, On the Synthesis of DOL Growth, to appear in Ann. Acad. Sci. Fenn., Serie A, I.

8. A. SalomaA, On Exponential Growth in Lindenmayer Systems, Indag. Math., vol. 35, 1973, pp. 23-30.

9. M. P. Schützenberger, On a Theorem of R. Jungen, Proc. Amer. Math. Soc., vol. 13, 1962, pp. 885-890.

10. P. VitanyI, Structure of Growth in Lindenmayer Systems, Indag. Math., vol. 35, 1973, pp. 247-253. 